\title{
Ecological informatics for ecosystem conservation in view of environmental risk assessment and management
}

\author{
Zhifeng Yang
}

Published online: 26 April 2011

(C) Springer-Verlag 2011

The present special issue of SERRA includes the contributions of scholars from all around the World who participated in the biennial International Conference on Ecological Informatics and Ecosystem Conservation (http://www.iseis.org/) held during August 27-29 in Beijing, China. This conference was organized by Beijing Normal University and the International Society for Environmental Information Sciences. The aim of the conference was to develop strategies to increase international access to the latest developments in ecological information sciences and ecosystem conservation and to strengthen collaborations among environmental and ecological scientists.

The collection of papers in this special issue is a fraction of those presented at the conference, which have been subjected to a rigorous review process. In general, these articles may be categorized into three groups:

(1) Environmental pollution and quality assessment

(2) Enviroinformatics for complex systems

(3) Ecological risk assessment and decision making

In the following, we shall briefly trace the main findings of the articles for each one of the above groups.

Group 1 includes three articles concerned with pollutants in atmosphere and soil, including environmental quality assessment and management. In terms of the chaos theory, $\mathrm{Yu}$ et al. established the phase space-reconstruction method to describe the multi-dimensional phase space for the time series of the air pollution index (API) during the last 10 years in Lanzhou, Northwest China. A few dynamic

\section{Z. Yang $(\bowtie)$}

State Key Laboratory of Water Environment Simulation,

School of Environment, Beijing Normal University,

Beijing 100875, China

e-mail: zfyang@bnu.edu.cn variables were also discussed that could effectively interpret temporal API changes and their causes. The method has been proved helpful for probing further into the regional complexity and evolution of the API time series. Nugroho et al. applied combined structural equation modeling and artificial neural networks to evaluate the impact of the bus rapid transit (BRT) system on the concentration of secondary pollutants in the roadside areas of the BRT corridors. An empirical analysis in the roadside areas of the TransJakarta corridors during 2005 indicated that the BRT system and the modal shift it produced had a greater influence on rapidly decaying pollutants (such as $\mathrm{PM}_{10}$ ) than on $\mathrm{O}_{3}$ due to exposure to near-source microenvironments. Bai et al. analyzed the contamination characteristics of heavy metals in wetland soils along a tidal ditch of the Yellow River Estuary, China. The results showed that (with the exception of As and $\mathrm{Cd}$ ) the mean concentrations of these heavy metals were lower than the class I criteria. Most of the heavy metals showed significant correlations with the total concentrations of $P$ and $S$; however, no significant correlations were observed between them and soil $\mathrm{pH}$, salt, and soil organic matter.

Group 2 consists of two articles on environmental informatics for complex systems. Yoshioka et al. introduced the energy service project that started in Tokyo in April 2010, which caps energy-related carbon dioxide emissions from 1330 offices and factories in Tokyo. The risk analysis and investment optimization for energy service projects was evaluated by using financial engineering. The results showed that the risk of energy service projects was spread under the Tokyo Emission Trading System. Zhang et al. employed network throughflow analysis and network utility analysis to probe into the structure of an urban energy metabolic system and the complex relationships among its components. Network throughflow analysis 
revealed that the energy transformation and consumption sectors had high weights during 4 years, whereas the energy recovery sector had small weights. The analysis of urban energy metabolic system provided guidance for optimizing the system's structure and adjusting the relationships among the sectors.

Group 3 includes three articles on ecological risk assessment, reliability analysis, and decision making. Qin et al. selected eight indices (biodiversity, biomass, and the uptake of $\mathrm{TN}, \mathrm{TP}, \mathrm{K}, \mathrm{Ca}, \mathrm{Mg}$, and $\mathrm{Na}$ ) at three scales (community, single plant, and micro-scale) to assess the ecological risks of water scarcity in the Yellow River delta based on the ecological value at risk model. A three-level classification of ecological risk was generated using Markov-Chain Monte Carlo (MCMC) techniques. The results could provide a foundation on which the planners allocate ecological flows and wetland restoration. $\mathrm{Xu}$ et al. proposed a research framework of urban ecological risk assessment, which combines the multimedia fugacity model, multi-route exposure model, exposure-risk relationships and geographic information systems (GIS). The urban ecological risk assessment of a hypothetical region indicated that it was feasible to introduce GIS into the previous method to satisfy the requirements of risk management. The assessment results could be used for industrial pollution emission control purposes. Cai et al. presented the development and the first application of an inexact quadratic programming (IQP) approach for sustainable water supply in conditions of multi-sourced uncertainty. The IQP approach improved conventional nonlinear programming by tackling multiple uncertainties within an individual parameter; IQP was also superior to the existing inexact methods due to its reflection of economies of scale and reduction of computational requirements. Corresponding to varied system conditions and decision priorities, the interval solutions from IQP could help generate a series of long-term water supply strategies that includes a number of economic, environmental, ecological, and water-security targets.

The Guest Editor would like to express his appreciation to the authors and reviewers for their great contributions to this special issue of SERRA. 